\title{
Optimal Tone Reservation for CDMA Systems
}

\author{
Holger Boche, Fellow, IEEE, and Ullrich J. Mönich, Senior Member, IEEE
}

\begin{abstract}
In this paper we analytically analyze the tone reservation method for the reduction of the peak to average power ratio (PAPR) in code division multiple access (CDMA) systems that employ the Walsh functions. We find the best possible reduction of the PAPR and give one optimal information set that achieves this reduction. Interestingly, when using more than one information carrier, the smallest possible extension constant is independent of the size of the information set and has the value $\sqrt{2}$. We further show that the minimal extension constant can also be achieved with finite compensation sets, and illustrate the findings with a numerical example. For certain special cases we are also able to provide results for the system of complex exponentials, which is employed in orthogonal frequency division multiplexing (OFDM).
\end{abstract}

Index Terms-Peak to average power, tone reservation, code division multiple access, Walsh system, optimal constant

\section{INTRODUCTION}

C ODE division multiple access (CDMA) is a transmission technique that is used in many systems, e.g., in $3 \mathrm{G}$ and UMTS, GPS, and Galileo [2]. Moreover, multiple extensions such as multicarrier CDMA [3] exist.

The control of the peak to average power ratio (PAPR) is an important task in any orthogonal transmission scheme that employs orthogonal functions [4]-[7]. Such orthogonal functions are used not only in CDMA, but also in other orthogonal transmission schemes, like orthogonal frequency division multiplexing (OFDM). Large PAPR values are undesirable, because they can overload amplifiers, distort the signals, and lead to out-of-band radiation. For a further discussion of these concepts and problems, we refer to [7].

Due to its importance, the PAPR problem has garnered much attention, and many different approaches to reduce the PAPR have been created [8]-[27]. In [14] and [18] new selected mapping approaches with reduced complexity were proposed. The selected mapping approach was further analyzed in [16], where the complementary cumulative distribution function performance was studied, and in [19], where an asymptotic performance analysis of selected mapping was performed. In [23] a PAPR reduction scheme that requires no side information was studied. A different constellation reshaping method was considered in [22].

Other publications consider PAPR reduction via optimization. An active-set minimax approach for tone reservation was analyzed in [12], and the application of convex optimization to calculate the OFDM signal with minimum PAPR was

This work was supported by the Gottfried Wilhelm Leibniz Programme of the German Research Foundation (DFG) under grant BO 1734/20-1.

The material in this paper was presented in part at the 2018 IEEE International Conference on Acoustics, Speech, and Signal Processing [1].

The authors are with the Technische Universität München, Lehrstuhl für Theoretische Informationstechnik, Munich 80290, Germany (e-mail: boche@tum.de; moenich@tum.de considered in [13]. Further, in [17], the adaptive projected subgradient method was studied.

In this paper we consider the popular tone reservation method [8], [9], [12], [21], [26]-[28]. In this method, the set of available carriers is partitioned into two sets, the information set $\mathcal{K}$, which is used to carry the information, and the compensation set $\mathcal{K}^{\complement}$, which is used to reduce the PAPR. A significant advantage of the tone reservation method is that no additional information exchange is needed between the transmitter and receiver. The set $\mathcal{K}$ is fixed and known by both parties. The receiver simply has to select the carriers corresponding to the set $\mathcal{K}$. We will explain the tone reservation method in more detail in Section III.

In [8] the tone reservation method for OFDM was introduced, and finding the best compensation signal was phrased as an optimization problem. However, the question of how to choose the compensation set was not treated, nor was an analytical analysis of the performance provided. A fastconverging active set method for finding the optimal compensation sequence was proposed in [12]. Then in [21], a first analytical approach for the analysis of the tone reservation method for OFDM was presented. There are further results for specific applications. The PAPR reduction in offset quadrature amplitude modulation-based orthogonal frequency division multiplexing was considered in [26], and a novel multi-block tone reservation scheme was proposed. Further, an adaptive tone reservation scheme for multi-user MIMO-OFDM systems that iteratively performs the tone reservation on the antenna with the highest PAPR was studied in [27]. While most of the above papers treat the PAPR problem for OFDM, the presented ideas can often be directly transferred to the PAPR problem for CDMA.

Reserving certain carriers for reducing the PAPR and not for transmitting information affects the energy efficiency of the entire communication system. Hence, from an energy perspective there is a trade-off, and to date, a thorough quantitative analysis of this trade-off seems to be missing for the tone reservation method. For PAPR reduction via partial transmit sequence, the energy-efficiency of non-contiguous OFDM offset quadrature amplitude modulation based radio networks was discussed in [24]. A first step towards the development of such an analysis for the tone reservation method, is to better understand it theoretically.

Tone reservation is an elegant procedure and easy to define. The practical implementation is difficult however, because there exist few explicit algorithms for the calculation of the compensation set, and their complexity is in general high. And even if one succeeded in finding an optimal compensation set-the exact meaning of which will become clear in Section IV-it is not easy to compute the specific compensation signal for a given information signal. Moreover, the 
performance of these algorithms is unclear, i.e., there are no performance guarantees and it is unknown how far they are from the theoretical optimum. Most available results are based on simulations and are not analytic.

In this paper we analytically treat the PAPR reduction problem via tone reservation for CDMA systems that employ the Walsh functions. For these CDMA systems we answer three important questions concerning the tone reservation method: 1 . What is the best possible reduction of the PAPR? 2. What is an optimal information set that achieves this reduction, and how can it be found? 3. What is the general structure of the optimal information sets? All questions are answered in Section IV. It is surprising that for CDMA, the optimal constants and information sets can be derived. In Section V we consider the practically important case of finite compensation sets and show that with a finite number of compensation functions the same optimal extension constant can be achieved as with infinitely many compensation functions. For certain special cases we are also able to provide results for OFDM. In particular, we derive the optimal OFDM extension constant for information sets of size two. The OFDM results are presented in Section VII.

Since the tone reservation method was studied by the authors also in several other publications [1], [29]-[31], some of the exposition in the introductory sections may overlap with those publications.

\section{NOTATION, CDMA, AND OFDM}

By $L^{p}[0,1], 1 \leq p \leq \infty$, we denote the usual $L^{p}$-spaces on the interval $[0,1]$, equipped with the norm $\|\cdot\|_{p}$. For an index set $\mathcal{I} \subset \mathbb{Z}$, we denote by $\ell^{2}(\mathcal{I})$ the set of all square summable sequences $c=\left\{c_{k}\right\}_{k \in \mathcal{I}}$ indexed by $\mathcal{I}$. The norm is given by $\|c\|_{\ell^{2}(\mathcal{I})}=\left(\sum_{k \in \mathcal{I}}\left|c_{k}\right|^{2}\right)^{1 / 2}$. By $|A|$ we denote the cardinality of a set $A$. For $N \in \mathbb{N}$ we use the abbreviation $[N]:=\{1,2, \ldots, N\}$.

The Rademacher functions $r_{n}, n \in \mathbb{N}$, on $[0,1]$ are defined by $r_{n}(t)=\operatorname{sgn}\left[\sin \left(\pi 2^{n} t\right)\right]$, where $\operatorname{sgn}$ denotes the signum function with the convention $\operatorname{sgn}(0)=-1$. The Walsh functions $w_{n}, n \in \mathbb{N}$, on $[0,1]$ are defined by

$$
w_{1}(t)=1
$$

and

$$
w_{2^{k}+m}(t)=r_{k+1}(t) w_{m}(t)
$$

for $k=0,1,2, \ldots$ and $m=1,2, \ldots, 2^{k}$. Note that we use an indexing of the Walsh functions that starts with 1 . The Rademacher system $\left\{r_{n}\right\}_{n \in \mathbb{N}}$ is an orthonormal system (ONS) in $L^{2}[0,1]$, but not a basis. The Walsh functions $\left\{w_{n}\right\}_{n \in \mathbb{N}}$ form an orthonormal basis for $L^{2}[0,1]$, and we have

$$
\int_{0}^{1} w_{n}(t) \mathrm{d} t=0
$$

for all $n \in \mathbb{N}$. For further details about the Walsh function, see, for example, [32].

The complete orthonormal system of Walsh functions $\left\{w_{n}\right\}_{n \in \mathbb{N}}$ is used in CDMA systems and will be the main subject of analysis in this paper. In OFDM systems, the employed set of functions is the set of complex exponentials $\left\{\mathrm{e}^{i 2 \pi k t}\right\}_{k \in \mathbb{Z}}$. Note that $\left\{\mathrm{e}^{i 2 \pi k t}\right\}_{k \in \mathbb{Z}}$ is a complete ONS in $L^{2}[0,1]$. We will briefly discuss the OFDM case in Section VII.

\section{PAPR AND TONE RESERVATION AND SOLVABILITY CONCEPTS}

Without loss of generality, we can restrict ourselves to signals defined on the interval $[0,1]$. Signals with other duration can be simply scaled to be concentrated on $[0,1]$. For a signal $s \in L^{2}[0,1]$, we define

$$
\operatorname{PAPR}(s)=\frac{\|s\|_{L^{\infty}[0,1]}}{\|s\|_{L^{2}[0,1]}},
$$

i.e., the PAPR is the ratio between the peak value of the signal and the square root of the power of the signal. Note that the PAPR is usually defined as the square of this value. However, from a mathematical point of view, this makes no difference for the results in this paper. In the case of an orthogonal transmission scheme, using the ONS $\left\{\phi_{k}\right\}_{k \in \mathcal{I}} \subset L^{2}[0,1]$, the PAPR of the transmit signal

$$
s(t)=\sum_{k \in \mathcal{I}} c_{k} \phi_{k}(t), \quad t \in[0,1]
$$

with coefficients $c=\left\{c_{k}\right\}_{k \in \mathcal{I}}$, is given by

$$
\operatorname{PAPR}(s)=\frac{\left\|\sum_{k \in \mathcal{I}} c_{k} \phi_{k}\right\|_{L^{\infty}[0,1]}}{\|c\|_{\ell^{2}(\mathcal{I})}},
$$

because $\left\{\phi_{k}\right\}_{k \in \mathcal{I}}$ is a ONS, which implies that $\|s\|_{L^{2}[0,1]}=$ $\|c\|_{\ell^{2}(\mathcal{I})}$.

For an orthogonal transmission scheme, the peak value of the signal $s$, and hence the PAPR, can become large, as the following result shows. Given any system $\left\{\phi_{n}\right\}_{n=1}^{N}$ of $N$ orthonormal functions in $L^{2}[0,1]$, then there exist a sequence $\left\{c_{n}\right\}_{n=1}^{N} \subset \mathbb{C}$ of coefficients with $\sum_{n=1}^{N}\left|c_{n}\right|^{2}=1$, such that $\left\|\sum_{n=1}^{N} c_{n} \phi_{n}\right\|_{L^{\infty}[0,1]} \geq \sqrt{N}$ [33]. This increase of the PAPR with an order of $\sqrt{N}$ is undesired and methods to battle it are needed.

The tone reservation method, which we will explain next, is one approach to reduce the PAPR. Let $\left\{\phi_{k}\right\}_{k \in \mathcal{I}}$ be an ONS in $L^{2}[0,1]$. We additionally assume that $\left\|\phi_{k}\right\|_{\infty}<\infty, k \in$ $\mathcal{I}$, i.e., we consider the practically relevant case of bounded carriers. In the tone reservation method, the index set $\mathcal{I}$ is partitioned in two disjoint sets $\mathcal{K}$ and $\mathcal{K}^{\complement}$. Note that the set $\mathcal{K}$ can be finite or infinite. For a given information sequence $a=\left\{a_{k}\right\}_{k \in \mathcal{K}} \in \ell^{2}(\mathcal{K})$, the goal is to find a compensation sequence $b=\left\{b_{k}\right\}_{k \in \mathcal{K}^{\mathrm{c}}} \in \ell^{2}\left(\mathcal{K}^{\mathrm{C}}\right)$ such that the peak value of the transmit signal

$$
s(t)=\underbrace{\sum_{k \in \mathcal{K}} a_{k} \phi_{k}(t)}_{=: A(t)}+\underbrace{\sum_{k \in \mathcal{K}^{\complement}} b_{k} \phi_{k}(t)}_{=: B(t)}, \quad t \in[0,1],
$$

is as small as possible. $A(t)$ denotes the signal part which contains the information and $B(t)$ the part which is used to reduce the PAPR.

A priori, it is not clear how to obtain the optimal sequence $b$ for a given information sequence $a$. In Section VI we will see that $b$ can be found by solving a convex optimization problem. For further details about the tone reservation method, we refer to [28].

A block diagram illustrating the tone reservation method for a CDMA transmission system using the Walsh functions 


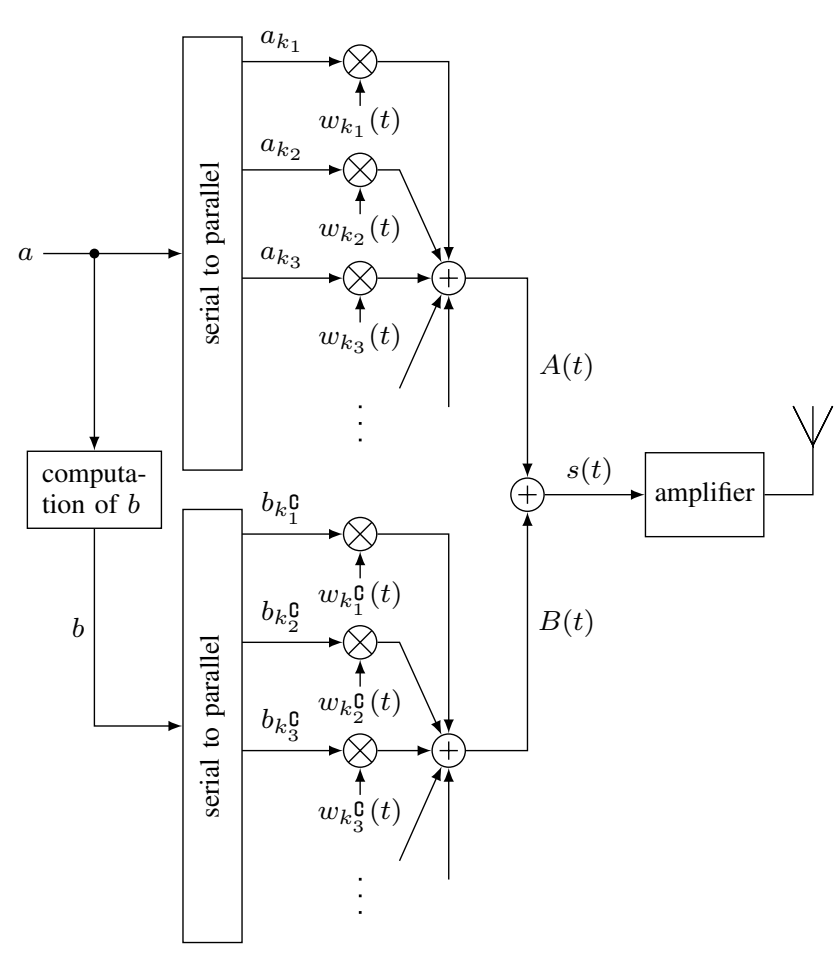

Fig. 1. Block diagram of a CDMA transmission scheme with tone reservation. In this example we have $\mathcal{I}=\mathbb{N}, \mathcal{K}=\left\{k_{1}, k_{2}, k_{3} \ldots\right\}$ and $\mathcal{K}^{\complement}=\mathbb{N} \backslash \mathcal{K}=$ $\left\{k_{1}^{\complement}, k_{2}^{\complement}, k_{3}^{\complement}, \ldots\right\}$. For OFDM we have the same structure. However, we then use the system of complex exponentials and the set $\mathcal{I}=\mathbb{Z}$.

is given in Fig. 1. For the Walsh functions, which we mainly consider in this paper, the index set is given by $\mathcal{I}=\mathbb{N}$. In Section VII, we also use the system of exponentials. Then the index set is $\mathcal{I}=\mathbb{Z}$.

Note that we allow infinitely many carriers to be used for the compensation of the PAPR. This is also of practical interest, since the solvability of the PAPR problem in this setting is a necessary condition for the solvability of the PAPR problem in the setting with finitely many carriers.

We next define the solvability of the PAPR problem.

Definition 1. For an ONS $\left\{\phi_{k}\right\}_{k \in \mathcal{I}}$ and a set $\mathcal{K} \subset \mathcal{I}$, we say that the PAPR problem is solvable with finite extension constant $C_{\mathrm{EX}}$, if for all $a \in \ell^{2}(\mathcal{K})$ there exists a $b \in \ell^{2}\left(\mathcal{K}^{\complement}\right)$ such that

$$
\left\|\sum_{k \in \mathcal{K}} a_{k} \phi_{k}+\sum_{k \in \mathcal{K}^{\complement}} b_{k} \phi_{k}\right\|_{L^{\infty}[0,1]} \leq C_{\mathrm{EX}}\|a\|_{\ell^{2}(\mathcal{K})} .
$$

We call the PAPR problem solvable if it is solvable for some finite extension constant $C_{\mathrm{EX}}$.

If the PAPR reduction problem is solvable, condition (1) immediately implies that $\|b\|_{\ell^{2}\left(\mathcal{K}^{\complement}\right)} \leq C_{\mathrm{EX}}\|a\|_{\ell^{2}(\mathcal{K})}$, because

$$
\begin{aligned}
\left(\sum_{k \in \mathcal{K}^{\complement}}\left|b_{k}\right|^{2}\right)^{\frac{1}{2}} & \leq\left(\sum_{k \in \mathcal{K}}\left|a_{k}\right|^{2}+\sum_{k \in \mathcal{K}^{\complement}}\left|b_{k}\right|^{2}\right)^{\frac{1}{2}} \\
& =\left(\int_{0}^{1}\left|\sum_{k \in \mathcal{K}} a_{k} \phi_{k}(t)+\sum_{k \in \mathcal{K}^{\complement}} b_{k} \phi_{k}(t)\right|^{2} \mathrm{~d} t\right)^{\frac{1}{2}}
\end{aligned}
$$

$$
\leq \operatorname{esssup}_{t \in[0,1]}\left|\sum_{k \in \mathcal{K}} a_{k} \phi_{k}(t)+\sum_{k \in \mathcal{K}^{\complement}} b_{k} \phi_{k}(t)\right|,
$$

that is, the energy of the compensation signal is also bounded by $\left(C_{\mathrm{EX}}\|a\|_{\ell^{2}(\mathcal{K})}\right)^{2}$. Further, we have $\operatorname{PAPR}(s) \leq C_{\mathrm{EX}}$. It is clear that finding the optimal, i.e., minimal extension constant is an important problem that is relevant for applications. Inequality (1) is also a statement about the compensation signals. However, (1) cannot be directly used for an analytical analysis. Therefore it is important to better understand the signals created by the permissible information sets. We will deal with this problem next. In [21], [34] the following different but equivalent characterization of solvability was given. In this characterization the set

$$
\begin{aligned}
\mathfrak{P}^{1}(\mathcal{K})=\left\{f \in L^{1}[0,1]:\right. & \\
& \left.f=\sum_{k \in \mathcal{K}} a_{k} \phi_{k} \text { for some }\left\{a_{k}\right\}_{k \in \mathcal{K}} \subset \mathbb{C}\right\}
\end{aligned}
$$

plays a central role.

Theorem 1. Let $\left\{\phi_{n}\right\}_{n \in \mathbb{N}}$ be a complete ONS, $\mathcal{K} \subset \mathbb{N}$, and $C_{E X}>0$. We have

$$
\|f\|_{L^{2}[0,1]} \leq C_{E X}\|f\|_{L^{1}[0,1]}
$$

for all $f \in \mathfrak{P}^{1}(\mathcal{K})$, if and only if the PAPR problem is solvable for $\left\{\phi_{n}\right\}_{n \in \mathbb{N}}$ and $\mathcal{K}$ with constant $C_{E X}$.

Remark 1. Theorem 1 is particularly interesting, because the characterization only depends on the information set $\mathcal{K}$ and the signals created by this set. That is, the compensation signals are irrelevant. This will help us in our further analysis.

Based on Theorem 1 we can establish the following important corollary.

Corollary 1. Let $\left\{\phi_{n}\right\}_{n \in \mathbb{N}}$ be a complete ONS and $\mathcal{K} \subset \mathbb{N}$ such that the PAPR problem is solvable, and set

$$
U=\left(\inf _{\substack{f \in \mathfrak{P}^{1}(\mathcal{K}) \\\|f\|_{L^{2}[0,1]}=1}}\|f\|_{L^{1}[0,1]}\right)^{-1} .
$$

Then the PAPR problem is solvable for $\left\{\phi_{n}\right\}_{n \in \mathbb{N}}$ and $\mathcal{K}$ with extension constant $C_{E X}=U$. Further, $U$ is the smallest of all possible extension constants.

Proof. We have

$$
\frac{1}{U}=\inf _{\substack{f \in \mathfrak{P}^{1}(\mathcal{K}) \\\|f\|_{L^{2}[0,1]}=1}}\|f\|_{L^{1}[0,1]}=\inf _{\substack{f \in \mathfrak{P}^{1}(\mathcal{K}) \\ f \neq 0}} \frac{\|f\|_{L^{1}[0,1]}}{\|f\|_{L^{2}[0,1]}} .
$$

Hence, we see that

$$
\|f\|_{L^{2}[0,1]} \leq U\|f\|_{L^{1}[0,1]}
$$

for all $f \in \mathfrak{P}^{1}(\mathcal{K})$. From Theorem 1 it follows that the PAPR problem is solvable with extension constant $C_{\mathrm{EX}}=U$. Next we prove that $U$ is the smallest possible extension constant. We do a proof by contradiction. Assume that there exists a smaller number $U_{1}<U$ such that the PAPR problem is solvable with 
extension constant $C_{\mathrm{EX}}=U_{1}$. Then we see from Theorem 1 that

$$
\|f\|_{L^{2}[0,1]} \leq U_{1}\|f\|_{L^{1}[0,1]}
$$

for all $f \in \mathfrak{P}^{1}(\mathcal{K})$. It follows that

$$
\frac{1}{U_{1}} \leq \inf _{\substack{f \in \mathfrak{P}^{1}(\mathcal{K}) \\\|f\|_{L^{2}[0,1]}=1}}\|f\|_{L^{1}[0,1]} .
$$

Using the definition of $U$ in (4), we obtain that

$$
\frac{1}{U_{1}} \leq \frac{1}{U}
$$

or equivalently $U_{1} \geq U$. However, this is a contradiction to our assumption.

Corollary 1 in particular implies that for any given information set $\mathcal{K} \subset \mathbb{N}$, under all extension constants $C_{\mathrm{EX}}$ for which the PAPR problem is solvable, there exists a smallest extension constant $\underline{C}_{\mathrm{EX}}(\mathcal{K})$. We call

$$
\underline{C}_{\mathrm{EX}}(\mathcal{K})=\left(\inf _{\substack{f \in \mathfrak{P}^{1}(\mathcal{K}) \\\|f\|_{L^{2}[0,1]}=1}}\|f\|_{L^{1}[0,1]}\right)^{-1}
$$

an optimal extension constant.

\section{OPTIMAL PAPR CONTROL AND OPTIMAL INFORMATION SETS FOR CDMA}

\section{A. Main Results}

For CDMA systems that employ the Walsh system as carrier functions $\left\{\phi_{n}\right\}_{n \in \mathbb{N}}=\left\{w_{n}\right\}_{n \in \mathbb{N}}$, we will next answer the three questions that were already stated in the introduction: 1 . What is the best possible reduction of the PAPR, i.e, how small is the optimal extension constant $C_{\mathrm{EX}}$ ? 2. What is an optimal information set $\mathcal{K}$ that achieves this reduction, and how can it be found? 3. What is the general structure of the optimal information sets? The proofs of all results in this section will be given in Section IV-D.

For a given information set $\mathcal{K} \subset \mathbb{N}$, we denote by $\underline{C}_{\mathrm{EX}}(\mathcal{K})$ the optimal, i.e., smallest, extension constant for which the PAPR problem is solvable for the Walsh system $\left\{\phi_{n}\right\}_{n \in \mathbb{N}}=$ $\left\{w_{n}\right\}_{n \in \mathbb{N}}$ and the set $\mathcal{K}$. Next, we want to study how small the optimal extension constant $\underline{C}_{\mathrm{EX}}(\mathcal{K})$, which was introduced in IV, can become for different sets $\mathcal{K}$ of cardinality $N$, i.e., we are interested in

$$
\underline{C}_{\mathrm{EX}}(N):=\inf _{\substack{\mathcal{K} \subset \mathbb{N} \\|\mathcal{K}|=N}} C_{\mathrm{EX}}(\mathcal{K}) .
$$

We will see in Theorem 4 that for each $N \in \mathbb{N}$ there indeed exists a set $\mathcal{K}_{\text {opt }}(N) \subset \mathbb{N}$ with $\left|\mathcal{K}_{\text {opt }}(N)\right|=N$, such that $\underline{C}_{\mathrm{EX}}(N)=\underline{C}_{\mathrm{EX}}\left(\left\{\mathcal{K}_{\text {opt }}(N)\right\}\right)$. That is, the infimum in (6) is in fact attained, and a minimum. Note that the set $\mathcal{K}_{\text {opt }}(N)$ does not need to be unique.

A priori, it is not clear how the set $\mathcal{K}_{\text {opt }}(N)$ depends on $N$. It could be that for different $N$ we obtain completely different sets $\mathcal{K}_{\text {opt }}(N)$.

The next theorem completely describes the smallest possible extension constant $\underline{C}_{\mathrm{EX}}$, and thus answers question 1 .
Theorem 2. We have $\underline{C}_{E X}(1)=1$ and $\underline{C}_{E X}(N)=\sqrt{2}$ for all $N \geq 2$.

Interestingly, for all $N \geq 2$ the smallest possible extension constant is independent of $N$. We call any information set $\mathcal{K} \subset \mathbb{N}$ that achieves the smallest possible extension constant an optimal information set.

For $N=2$ we have the important result that all possible information sets $\mathcal{K} \subset \mathbb{N}$ with $|\mathcal{K}|=2$ are optimal, i.e., give the smallest possible extension constant.

Theorem 3. For all $k_{1}, k_{2} \in \mathbb{N}, k_{1} \neq k_{2}$, we have $\underline{C}_{E X}\left(\left\{k_{1}, k_{2}\right\}\right)=\sqrt{2}$.

The general case for arbitrary $N \in \mathbb{N}$ is given next. This answers question 2 about finding an optimal information set $\mathcal{K}_{\text {opt }}(N)$ that achieves the best possible PAPR reduction.

Theorem 4. For $N \in \mathbb{N}$ we have $\underline{C}_{E X}(N)=\underline{C}_{E X}\left(\left\{2^{k}+\right.\right.$ $\left.1\}_{k=0}^{N-1}\right)$. That is, we have $\mathcal{K}_{\text {opt }}(N)=\left\{2^{k}+1\right\}_{k=0}^{N-1}$, i.e., the first $N$ Rademacher functions achieve the minimal extension constant $\underline{C}_{E X}(N)$.

Remark 2. Theorem 4 shows that there exists a universal infinite information set $\mathcal{K}=\left\{2^{k}+1\right\}_{k=0}^{\infty}$ such that for every $N \geq 2$, each subset $\mathcal{K}_{N}=\left\{2^{k}+1\right\}_{k=0}^{N-1}$ consisting of the smallest $N$ elements of $\mathcal{K}$, already achieves the smallest possible extension constant $\underline{C}_{\mathrm{EX}}\left(\mathcal{K}_{N}\right)=\sqrt{2}$. Note that the set $\mathcal{K}=\left\{2^{k}+1\right\}_{k=0}^{\infty}$ itself also satisfies $\underline{C}_{\mathrm{EX}}(\mathcal{K})=\sqrt{2}$.

Finally, we study the structure of the optimal information sets, and thus answer question 3 . To this end, we introduce

$$
T_{\text {opt }}=\left\{\mathcal{K} \subset \mathbb{N}:|\mathcal{K}| \geq 2, \underline{C}_{\mathrm{EX}}(\mathcal{K})=\sqrt{2}\right\},
$$

which contains all optimal information sets $\mathcal{K} \subset \mathbb{N}$ of arbitrary cardinality greater than or equal to 2 . We will now show that for every set $\mathcal{K} \in T_{\text {opt }}$ we have: Any subset of $\mathcal{K}$ gives the optimal extension constant. This follows from the next theorem, for which we need to introduce the set

$$
\begin{aligned}
& \mathfrak{W}^{1}(\mathcal{K})=\left\{f \in L^{1}[0,1]:\right. \\
& \left.\quad f=\sum_{k \in \mathcal{K}} a_{k} w_{k} \text { for some }\left\{a_{k}\right\}_{k \in \mathcal{K}} \subset \mathbb{C}\right\} .
\end{aligned}
$$

Theorem 5. Let $\mathcal{K} \subset \mathbb{N}$ with $|\mathcal{K}| \geq 2$ be arbitrary. Then $\mathcal{K}$ is optimal, i.e. $\underline{C}_{E X}(\mathcal{K})=\sqrt{2}$, if and only if for arbitrary sets of subsets

$$
\mathcal{K}_{1} \subset \mathcal{K}_{2} \subset \cdots \subset \mathcal{K}
$$

where $\left|\mathcal{K}_{1}\right|=2$, we have

$$
\inf _{\substack{f \in \mathfrak{W}^{1}\left(\mathcal{K}_{1}\right) \\\|f\|_{L^{2}[0,1]}=1}}\|f\|_{L^{1}[0,1]}=\cdots=\inf _{\substack{f \in \mathfrak{W}^{1}(\mathcal{K}) \\\|f\|_{L^{2}[0,1]}=1}}\|f\|_{L^{1}[0,1]} .
$$

An immediate consequence of Theorem 5 is that subsets of optimal information sets are always optimal.

Corollary 2. Let $\mathcal{K} \in T_{\text {opt }}$ be an optimal information set. Then any subset $\mathcal{K}_{1} \subset \mathcal{K}$ with $\left|\mathcal{K}_{1}\right| \geq 2$ is optimal as well, i.e., we have $\mathcal{K}_{1} \in T_{\text {opt }}$.

Of course we cannot conclude that for $\mathcal{K} \in T_{\text {opt }}$ and $k_{l} \notin \mathcal{K}$, we have $\mathcal{K} \cup\left\{k_{l}\right\} \in T_{\text {opt }}$. 


\section{B. Elementary Facts About Walsh Functions}

Before we can give the proofs we need some elementary facts about Walsh functions which were introduced in Section II.

Let $\mathcal{K}=\left\{k_{1}, k_{2}, \ldots, k_{N}\right\} \subset \mathbb{N}$ be a set of $N$ arbitrary distinct natural numbers. Further, let $W(\mathcal{K})$ denote the largest number $C_{1}$ such that

$$
C_{1}\left(\sum_{l=1}^{N}\left|\alpha_{l}\right|^{2}\right)^{\frac{1}{2}} \leq \int_{0}^{1}\left|\sum_{l=1}^{N} \alpha_{l} w_{k_{l}}(t)\right| \mathrm{d} t
$$

for all $\alpha_{1}, \ldots, \alpha_{N} \in \mathbb{C}$. Then we have

$$
\begin{aligned}
W(\mathcal{K})= & \inf _{\substack{\left\{\alpha_{l}\right\}_{l=1}^{N} \\
\sum_{l=1}^{N}\left|\alpha_{l}\right|^{2}=1}} \int_{0}^{1}\left|\sum_{l=1}^{N} \alpha_{l} \omega_{k_{l}}(t)\right| \mathrm{d} t \\
= & \min _{\substack{\left\{\alpha_{l}\right\}_{l=1}^{N} \\
\sum_{l=1}^{N}\left|\alpha_{l}\right|^{2}=1}} \int_{0}^{1}\left|\sum_{l=1}^{N} \alpha_{l} \omega_{k_{l}}(t)\right| \mathrm{d} t .
\end{aligned}
$$

The minimum in (10) is indeed attained, since the mapping

$$
\left(a_{1}, \ldots, a_{N}\right) \mapsto \int_{0}^{1}\left|\sum_{l=1}^{N} \alpha_{l} \omega_{k_{l}}(t)\right| \mathrm{d} t
$$

is continuous, and the minimum is taken over a compact set in $\mathbb{C}^{N}$. According to the Cauchy-Schwarz inequality we have

$$
\begin{aligned}
\int_{0}^{1}\left|\sum_{l=1}^{N} \alpha_{l} w_{k_{l}}(t)\right| \mathrm{d} t & \leq\left(\int_{0}^{1}\left|\sum_{l=1}^{N} \alpha_{l} w_{k_{l}}(t)\right|^{2} \mathrm{~d} t\right)^{\frac{1}{2}} \\
& =\left(\sum_{l=1}^{N}\left|\alpha_{l}\right|^{2}\right)^{\frac{1}{2}},
\end{aligned}
$$

which implies that

$$
W(\mathcal{K}) \leq 1 .
$$

Further, for arbitrary $l \in[N]$, we see from (10) that

$$
W(\mathcal{K}) \leq W\left(\mathcal{K} \backslash\left\{k_{l}\right\}\right) .
$$

For $N \in \mathbb{N}$ we set

$$
\bar{W}(N):=\sup _{\substack{\mathcal{K} \subset \mathbb{N} \\|\mathcal{K}| \leq N}} W(\mathcal{K}) .
$$

Clearly, we have $0 \leq \bar{W}(N) \leq 1$ for all $N \in \mathbb{N}$, according to (11). For $N \geq 2$ and all sets $\left\{k_{1}, \ldots, k_{N}\right\}$, it follows from (12) that

$$
W\left(\left\{k_{1}, \ldots, k_{N}\right\}\right) \leq \bar{W}(N-1),
$$

and consequently

$$
\bar{W}(N) \leq \bar{W}(N-1) .
$$

Hence, we see that $0 \leq \bar{W}(N) \leq 1$ and, further, that $\{\bar{W}(N)\}_{N=1}^{\infty}$ is a monotonically decreasing sequence of real numbers that is bounded from below. Hence the limit $\lim _{N \rightarrow \infty} \bar{W}(N)$ exists.

\section{Auxiliary Result About Rademacher Functions}

In this section we prove an auxiliary result about Rademacher functions that will be needed for the proof of our main results.

For $N \in \mathbb{N}$, let $R(N)$ denote the largest number $C_{2}$ such that

$$
C_{2}\left(\sum_{l=1}^{N}\left|\alpha_{l}\right|^{2}\right)^{\frac{1}{2}} \leq \int_{0}^{1}\left|\sum_{l=1}^{N} \alpha_{l} r_{l}(t)\right| \mathrm{d} t
$$

for all $\alpha_{1}, \ldots, \alpha_{N} \in \mathbb{C}$. By the same reasoning as in Section IV-B, there exists such a number.

Lemma 1. We have $R(1)=1$ and $R(N)=1 / \sqrt{2}$ for all $N \geq 2$.

Proof. For $N=1$, eq. (14) becomes $C_{2}\left|\alpha_{1}\right| \leq\left|\alpha_{1}\right|$, which shows that $R(1)=1$.

Next, we treat the case $N \geq 2$. According to Khinchin's inequality, we have for all $N \in \mathbb{N}$ and all $\alpha_{1}, \ldots, \alpha_{N} \in \mathbb{C}$ that

$$
\frac{1}{\sqrt{2}}\left(\sum_{l=1}^{N}\left|\alpha_{l}\right|^{2}\right)^{\frac{1}{2}} \leq \int_{0}^{1}\left|\sum_{l=1}^{N} \alpha_{l} r_{l}(t)\right| \mathrm{d} t .
$$

The constant $1 / \sqrt{2}$ in (15) is the best, i.e., largest possible constant that holds for all $N \in N$ [35], [36]. For fixed $N \in \mathbb{N}$, $N \geq 2$, inequality (15) implies that

$$
R(N) \geq \frac{1}{\sqrt{2}}
$$

A simple calculation shows that

$$
\int_{0}^{1}\left|r_{1}(t)+r_{2}(t)\right| \mathrm{d} t=1
$$

Hence, for $N=2$ and $\alpha_{1}=\alpha_{1}=1$, inequality (14) becomes $C_{2} \sqrt{2} \leq 1$, and and we see that $R(2) \leq 1 / \sqrt{2}$. Due to (16), it follows that

$$
R(2)=\frac{1}{\sqrt{2}} .
$$

Further, for $N \geq 3$ we have

$$
\frac{1}{\sqrt{2}} \leq R(N) \leq R(2) \leq \frac{1}{\sqrt{2}}
$$

where the first inequality follows from (16), the second inequality from the same arguments that led to (13), and the third inequality from (17). Hence, for $N \geq 2$, we have $R(N)=1 / \sqrt{2}$.

\section{Proofs}

In this section we prove the results that were presented in Section IV-A.

Based on Lemma 1 we can prove Lemma 2, which is needed for the proof of Theorem 2.

Lemma 2. We have $\bar{W}(1)=1$ and $\bar{W}(N)=1 / \sqrt{2}$ for all $N \geq 2$.

Proof. Since

$$
W(\{k\})=\int_{0}^{1}\left|w_{k}(t)\right| \mathrm{d} t=1,
$$


for all $k \in \mathbb{N}$, it immediately follows that $\bar{W}(1)=1$. According to the definition of $\bar{W}$, we have $\bar{W}(N) \geq R(N)$ for all $N \in \mathbb{N}$. Hence, it follows from Lemma 1 that

$$
\bar{W}(N) \geq \frac{1}{\sqrt{2}}
$$

for all $N \geq 2$. Let $k_{1}<k_{2}$ be two arbitrary natural numbers. We have

$$
\begin{aligned}
\int_{0}^{1}\left|w_{k_{1}}(t)+w_{k_{2}}(t)\right| \mathrm{d} t & =\int_{0}^{1}\left|w_{k_{1}}(t)\left(w_{k_{1}}(t)+w_{k_{2}}(t)\right)\right| \mathrm{d} t \\
& =\int_{0}^{1}\left|1+w_{k_{1}}(t) w_{k_{2}}(t)\right| \mathrm{d} t \\
& =\int_{0}^{1}\left|1+w_{k^{\prime}}(t)\right| \mathrm{d} t \\
& =\int_{0}^{1} 1+w_{k^{\prime}}(t) \mathrm{d} t \\
& =1
\end{aligned}
$$

and it follows that

$$
\begin{aligned}
W\left(\left\{k_{1}, k_{2}\right\}\right) & \leq \int_{0}^{1}\left|\frac{1}{\sqrt{2}} w_{k_{1}}(t)+\frac{1}{\sqrt{2}} w_{k_{2}}(t)\right| \mathrm{d} t \\
& =\frac{1}{\sqrt{2}},
\end{aligned}
$$

where we used (10) in the inequality and (19) in the equality. Hence, we see that

$$
\bar{W}(2) \leq \frac{1}{\sqrt{2}}
$$

Since $\bar{W}(N) \leq \bar{W}(2)$ for all natural numbers $N \geq 2$ according to (13), it follows that

$$
\frac{1}{\sqrt{2}} \leq \bar{W}(N) \leq \bar{W}(2) \leq \frac{1}{\sqrt{2}},
$$

where we used (18) in the first and (21) in the last inequality. Consequently, we have $\bar{W}(N)=1 / \sqrt{2}$ for all $N \geq 2$.

Now we are in the position to prove Theorems 2 and 4 .

Proof of Theorem 2. According to the definitions of $C_{\mathrm{EX}}(N)$ and $\bar{W}(N)$, and by using Theorem 1 , we see that $C_{\mathrm{EX}}(N)=$ $1 / \bar{W}(N)$. Hence, Lemma 2 completes the proof.

Proof of Theorem 3. From (20) we know that $W\left(\left\{k_{1}, k_{2}\right\}\right) \leq$ $1 / \sqrt{2}$. Since $W\left(\left\{k_{1}, k_{2}\right\}\right) \leq \bar{W}(2)=1 / \sqrt{2}$, where the inequality follows from the definition of $\bar{W}$ and the equality from Lemma 2, we see that $W\left(\left\{k_{1}, k_{2}\right\}\right)=1 / \sqrt{2}$. Using

$$
\underline{C}_{\mathrm{EX}}\left(\left\{k_{1}, k_{2}\right\}\right)=\frac{1}{W\left(\left\{k_{1}, k_{2}\right\}\right)},
$$

which follows from (5) and (10), we finally obtain $\underline{C}_{\mathrm{EX}}\left(\left\{k_{1}, k_{2}\right\}\right)=\sqrt{2}$.

Proof of Theorem 4. In the proof of Lemma 1 we have already seen that for every $N \in \mathbb{N}$, the first $N$ Rademacher functions $r_{1}, \ldots, r_{N}$ give the maximal constant $\bar{W}(N)$ and hence the minimal extension constant $C_{\mathrm{EX}}(N)$. The first $N$ Rademacher functions $\left\{r_{n}\right\}_{n=1}^{N}$ correspond to the Walsh functions $\left\{w_{2^{k}+1}\right\}_{k=0}^{N-1}$.
Proof of Theorem 5. "£": From Theorem 3 we know that

$$
\inf _{\substack{f \in \mathfrak{W}^{1}\left(\mathcal{K}_{1}\right) \\\|f\|_{L^{2}[0,1]}=1}}\|f\|_{L^{1}[0,1]}=\frac{1}{\sqrt{2}} .
$$

Hence, from (8) it follows that

$$
\inf _{\substack{f \in \mathfrak{W}^{1}(\mathcal{K}) \\\|f\|_{L^{2}[0,1]}=1}}\|f\|_{L^{1}[0,1]}=\frac{1}{\sqrt{2}}
$$

which in turn, using (5), implies that $\underline{C}_{\mathrm{EX}}(\mathcal{K})=\sqrt{2}$.

" $\Rightarrow$ ": Let $\mathcal{K}$ be optimal, i.e., $\underline{C}_{\mathrm{EX}}(\mathcal{K})=\sqrt{2}$, and let $\mathcal{K}_{1}, \mathcal{K}_{2}, \ldots$ be sets satisfying (7). Due to (7), we have

$$
\inf _{\substack{f \in \mathfrak{W}^{1}\left(\mathcal{K}_{1}\right) \\\|f\|_{L^{2}[0,1]}=1}}\|f\|_{L^{1}[0,1]} \geq \cdots \geq \inf _{\substack{f \in \mathfrak{W}^{1}(\mathcal{K}) \\\|f\|_{L^{2}[0,1]}=1}}\|f\|_{L^{1}[0,1]} .
$$

Since $\underline{C}_{\mathrm{EX}}(\mathcal{K})=\sqrt{2}$, according to our assumption, we have

$$
\inf _{\substack{f \in \mathfrak{W}^{1}(\mathcal{K}) \\\|f\|_{L^{2}[0,1]}=1}}\|f\|_{L^{1}[0,1]}=\frac{1}{\sqrt{2}},
$$

due to (5). Further, we have

$$
\inf _{\substack{f \in \mathfrak{W}^{1}\left(\mathcal{K}_{1}\right) \\\|f\|_{L^{2}[0,1]}=1}}\|f\|_{L^{1}[0,1]}=\frac{1}{\sqrt{2}},
$$

according to Theorem 3. Inequality (23) together with (24) and (25) implies (8).

Proof of Corollary 2. According to Theorem 5, we have

$$
\inf _{\substack{f \in \mathfrak{W}^{1}\left(\mathcal{K}_{1}\right) \\\|f\|_{L^{2}[0,1]}=1}}\|f\|_{L^{1}[0,1]}=\frac{1}{\sqrt{2}} .
$$

The assertion follows from (5).

\section{Finite Compens ation Sets}

So far our analysis has been for infinite compensation sets. Next, we analyze the behavior of tone reservation for certain finite compensation sets. For information sets $\mathcal{K} \subset \mathbb{N}$, we use the abbreviation $\mathcal{K}_{N}=\mathcal{K} \cap\left[2^{N}\right]$. Now we consider the finite compensation set $\mathcal{K}_{N}^{\mathrm{Cf}}=\left[2^{N}\right] \backslash \mathcal{K}_{N}$.

Let $\underline{C}_{\mathrm{EX}}\left(\mathcal{K}_{N}, 2^{N}\right)$ be the smallest number such that for all $a \in \ell^{2}\left(\mathcal{K}_{N}\right)$ we can find a $b \in \ell^{2}\left(\mathcal{K}_{N}^{\mathrm{Cf}}\right)$ such that

$\left\|\sum_{k \in \mathcal{K}_{N}} a_{k} w_{k}+\sum_{k \in \mathcal{K}_{N}^{\mathrm{Cf}}} b_{k} w_{k}\right\|_{L^{\infty}[0,1]} \leq \underline{C}_{\mathrm{EX}}\left(\mathcal{K}_{N}, 2^{N}\right)\|a\|_{\ell^{2}\left(\mathcal{K}_{N}\right)}$.

That is to say, $\underline{C}_{\mathrm{EX}}\left(\mathcal{K}_{N}, 2^{N}\right)$ is the smallest extension constant for which the PAPR problem is solvable with a finite compensation set $\mathcal{K}_{N}^{\mathrm{Cf}}$.

Theorem 6. Let $\mathcal{K} \subset \mathbb{N}$ be an optimal information set and $N \in \mathbb{N}$ arbitrary with $\left|\mathcal{K}_{N}\right|>1$. Then we have

$$
\underline{C}_{E X}\left(\mathcal{K}_{N}, 2^{N}\right)=\sqrt{2} \text {. }
$$

Theorem 6 shows that using finite compensation sets is already as good as using infinite sets, because the same extension constant $\sqrt{2}$ can be achieved. 
Proof. Since we are restricting the compensation set, we have $\underline{C}_{\mathrm{EX}}\left(\mathcal{K}_{N}, 2^{N}\right) \geq \sqrt{2}$. Since $\mathcal{K}$ is an optimal information set, we know from Corollary 2 that $\mathcal{K}_{N}$ is an optimal information set. Hence for arbitrary $a \in \ell^{2}\left(\mathcal{K}_{N}\right)$ there exists a $b \in \ell^{2}(\mathbb{N} \backslash$ $\left.\mathcal{K}_{N}\right)$ such that

$$
\left\|\sum_{k \in \mathcal{K}_{N}} a_{k} w_{k}+\sum_{k \in \mathbb{N} \backslash \mathcal{K}_{N}} b_{k} w_{k}\right\|_{L^{\infty}[0,1]} \leq \sqrt{2}\|a\|_{\ell^{2}\left(\mathcal{K}_{N}\right)} .
$$

We consider

$$
F(t)=\sum_{k \in \mathcal{K}_{N}} a_{k} w_{k}(t)+\sum_{k \in \mathbb{N} \backslash \mathcal{K}_{N}} b_{k} w_{k}(t)=\sum_{k \in \mathbb{N}} \alpha_{k} w_{k}(t)
$$

and

$$
F_{N}(t)=\sum_{k=1}^{2^{N}} \alpha_{k} w_{k}(t)=\int_{0}^{1} F(\tau) P_{N}(t, \tau) \mathrm{d} \tau,
$$

where

$$
P_{N}(t, \tau)=\sum_{l=1}^{2^{N}} w_{l}(t) w_{l}(\tau) .
$$

We have $P_{N}(t, \tau) \geq 0$ for all $(t, \tau) \in[0,1]^{2}$ and

$$
\int_{0}^{1} P_{N}(t, \tau) \mathrm{d} \tau=1
$$

for all $t \in[0,1]$. It follows that

$$
\begin{aligned}
\left|F_{N}(t)\right| & \leq \int_{0}^{1}|F(\tau)| P_{N}(t, \tau) \mathrm{d} \tau \\
& \leq\|F\|_{L^{\infty}[0,1]} \\
& \leq \sqrt{2}\|a\|_{\ell^{2}(\mathcal{K})} .
\end{aligned}
$$

Hence, we see that

$$
\left\|F_{N}\right\|_{L^{\infty}[0,1]} \leq \sqrt{2}\|a\|_{\ell^{2}(\mathcal{K})},
$$

and consequently that $\underline{C}_{\mathrm{EX}}\left(\mathcal{K}_{N}, 2^{N}\right) \leq \sqrt{2}$.

\section{A. Structural Properties}

For the finite information sets $\mathcal{K}_{N}$, we can further characterize the structure. We will see that these sets cannot contain perfect Walsh sums if they are supposed to be optimal.

Let $N \in \mathbb{N}$ be arbitrary. For a set $\mathcal{S} \subset\left[2^{N}\right]$, we call the sum

$$
\sum_{s \in \mathcal{S}} w_{s}(t)
$$

a perfect Walsh sum, if there exists mutually distinct natural numbers $k_{1}, k_{2}, \ldots, k_{r} \in\left[2^{N}\right]$ such that

$\sum_{s \in \mathcal{S}} w_{s}(t)=w_{k_{1}}(t)\left(1+w_{k_{2}}(t)\right) \cdots \cdot\left(1+w_{k_{r+1}}(t)\right), \quad t \in[0,1]$

In this case, we call $S$ a perfect Walsh set and $|S|$ the size of the perfect Walsh sum. If $\mathcal{S}$ is a perfect Walsh set, then we have $2^{r}=|S|$. We have the following combinatoric condition for optimal information sets.

Theorem 7. Let $\mathcal{K} \subset \mathbb{N}$ be an optimal information set and $N \in \mathbb{N}$ arbitrary with $\left|\mathcal{K}_{N}\right|>1$. Then $\mathcal{K}_{N}$ does not contain a perfect Walsh set of size $|\mathcal{S}|>2$.

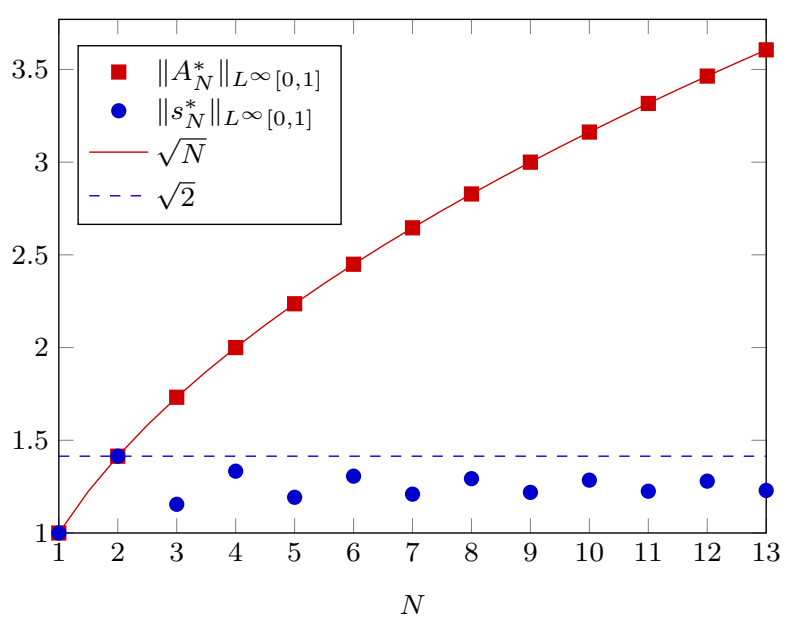

Fig. 2. Plot of the $L^{\infty}[0,1]$-norm of the uncompensated information signal $A_{N}^{*}$ (red) and the transmit signal $s_{N}^{*}$ (blue) for $N=1, \ldots, 13$. The $L^{\infty}[0,1]$-norm of the uncompensated information signal $A_{N}^{*}$ shows the worst-case $\sqrt{N}$ behavior. The $L^{\infty}[0,1]$-norm of the compensated information signal, i.e., the transmit signal $s_{N}^{*}$, is smaller than or equal to $\sqrt{2}$ for all $N$.

Remark 3. $\mathcal{K}_{N}$ always contains a perfect Walsh set of size 2 .

We conjecture that $\mathcal{K}_{N}$ also needs to be sparse in $\left[2^{N}\right]$.

Proof. We use a proof by contradiction. Assume that $\mathcal{K}_{N}$ contains a perfect Walsh set of size $|\mathcal{S}|>2$. Then we have

$$
\sum_{s \in \mathcal{S}} w_{s}(t)=w_{k_{1}}(t)\left(1+w_{k_{2}}(t)\right) \cdot \ldots \cdot\left(1+w_{k_{1+\log _{2}|\mathcal{S}|}}(t)\right)
$$

and

$$
\begin{aligned}
& \int_{0}^{1}\left|\sum_{s \in \mathcal{S}} w_{\mathcal{S}}(t)\right| \mathrm{d} t \\
& =\int_{0}^{1}\left|w_{k_{1}}(t)\left(1+w_{k_{2}}(t)\right) \cdot \ldots \cdot\left(1+w_{k_{1+\log _{2}|\mathcal{S}|}}(t)\right)\right| \mathrm{d} t \\
& =\int_{0}^{1}\left(1+w_{k_{2}}(t)\right) \cdot \ldots \cdot\left(1+w_{k_{1+\log _{2}|\mathcal{S}|}}(t)\right) \mathrm{d} t \\
& =1 \text {. }
\end{aligned}
$$

Since $\mathcal{S} \subset \mathcal{K}$, it follows from Corollary 2 that $\mathcal{S}$ is an optimal information set. Hence, we have

$$
\left(\int_{0}^{1}\left|\sum_{s \in \mathcal{S}} w_{s}(t)\right|^{2} \mathrm{~d} t\right)^{\frac{1}{2}} \leq \sqrt{2} \int_{0}^{1}\left|\sum_{s \in \mathcal{S}} w_{s}(t)\right| \mathrm{d} t
$$

according to Theorem 1. Using (27) and the fact that

$$
\left(\int_{0}^{1}\left|\sum_{s \in \mathcal{S}} w_{s}(t)\right|^{2} \mathrm{~d} t\right)^{\frac{1}{2}}=\sqrt{|S|}
$$

we see that $\sqrt{|S|} \leq \sqrt{2}$, which is a contradiction to our assumption $|\mathcal{S}|>2$.

\section{Optimization Perspective and Simulation}

In this section we will illustrate the findings with a simulation and use the results of the previous sections to compute the optimal compensation sequence $b$. In particular, we will see 
that it is sufficient to consider finite compensation sequences. Further, we argue that the optimal $b$ can be found by solving a convex optimization problem.

For our simulation, we consider different numbers of information carriers $N \in \mathbb{N}$. For a given $N$, we choose $\mathcal{K}_{N}=$ $\mathcal{K}_{\text {opt }}(N)=\left\{2^{k}+1\right\}_{k=0}^{N-1}$, i.e., an optimal information set. Further, we set $a_{k}^{*}=1 / \sqrt{N}, k \in \mathcal{K}_{N}$. Thus, the information sequence $\left\{a_{k}^{*}\right\}_{k \in \mathcal{K}_{N}}$ satisfies $\left\|\left\{a_{k}^{*}\right\}_{k \in \mathcal{K}_{N}}\right\|_{\ell^{2}\left(\mathcal{K}_{N}\right)}=1$, and the information signal

$$
A_{N}^{*}(t)=\sum_{k \in \mathcal{K}_{N}} a_{k}^{*} w_{k}(t)
$$

attains the largest possible peak value of $\left\|A_{N}^{*}\right\|_{L^{\infty}[0,1]}=\sqrt{N}$, as shown in Figure 2 in red. By using compensation carriers we can reduce the peak value. According to Theorem 6 , it is sufficient to consider the compensation set $\mathcal{K}_{N}^{\mathrm{Cf}}=\left[2^{N}\right] \backslash \mathcal{K}_{N}$ for an optimal compensation. The goal is to find the optimal compensation sequence $\left\{b_{k}\right\}_{k \in \mathcal{K}_{N}^{\mathrm{Cf}}} \in \ell^{2}\left(\mathcal{K}_{N}^{\mathrm{Cf}}\right)$ that minimizes the peak value of the transmit signal

$$
s_{N}(t)=\sum_{k \in \mathcal{K}_{N}} a_{k}^{*} w_{k}(t)+\sum_{k \in \mathcal{K}_{N}^{\mathrm{Cf}}} b_{k} w_{k}(t),
$$

i.e., we are interested in

$$
\left\{b_{k}^{*}\right\}_{k \in \mathcal{K}_{N}^{\mathrm{Cf}}}=\underset{b \in \ell^{2}\left(\mathcal{K}_{N}^{\mathrm{Cf}}\right)}{\arg \min }\left\|\sum_{k \in \mathcal{K}_{N}} a_{k}^{*} w_{k}+\sum_{k \in \mathcal{K}_{N}^{\mathrm{Cf}}} b_{k} w_{k}\right\|_{L^{\infty}[0,1]} .
$$

We first argue that this optimization problem has a solution. It can be easily seen that the function to be minimized is convex due to the properties of the norm. Further, the feasible set is convex. Thus, for fixed information set $\mathcal{K}_{N}$ and fixed information sequence $\left\{a_{k}^{*}\right\}_{k \in \mathcal{K}_{N}}$, we have a convex optimization problem. Since the feasible set in (28), i.e., $\ell^{2}\left(\mathcal{K}_{N}^{\mathrm{Cf}}\right)$, is unbounded, it is a priori unclear whether the minimization problem has always a solution. However, as we will show next, in our setting, the feasible set is in fact a compact set, and hence the minimization problem has always a solution. According to Theorem 6, we have

$$
\underline{C}_{\mathrm{EX}}\left(\mathcal{K}_{N}, 2^{N}\right)=\sqrt{2}
$$

and therefore it follows that there exists a $\tilde{b} \in \ell^{2}\left(\mathcal{K}_{N}^{\mathrm{Cf}}\right)$ such that

$$
\left\|\sum_{k \in \mathcal{K}_{N}} a_{k}^{*} w_{k}+\sum_{k \in \mathcal{K}_{N}^{\mathrm{Cf}}} \tilde{b}_{k} w_{k}\right\|_{L^{\infty}[0,1]} \leq \sqrt{2} .
$$

Hence, it suffices to restrict the feasible set in the minimization problem to sequences $b \in \ell^{2}\left(\mathcal{K}_{N}^{\mathrm{Cf}}\right)$ that satisfy (29). From (29) it follows that

$$
\left\|\sum_{k \in \mathcal{K}_{N}} a_{k}^{*} w_{k}+\sum_{k \in \mathcal{K}_{N}^{\mathrm{Cf}}} b_{k} w_{k}\right\|_{L^{2}[0,1]} \leq \sqrt{2}
$$

and consequently that

$$
\left\|\left\{b_{k}\right\}_{k \in \mathcal{K}_{N}^{\mathrm{Cf}}}\right\|_{\ell^{2}\left(\mathcal{K}_{N}^{\mathrm{Cf}}\right)} \leq \sqrt{2}
$$

because

$$
\begin{aligned}
& \left.\left\|\left\{b_{k}\right\}_{k \in \mathcal{K}_{N}^{\mathrm{Cf}}}\right\|_{\ell^{2}\left(\mathcal{K}_{N}^{\mathrm{Cf}}\right)}=\left\|\sum_{k \in \mathcal{K}_{N}^{\mathrm{Cf}}} b_{k} w_{k}\right\|_{L^{2}[0,1]}+\left\|\sum_{k \in \mathcal{K}_{N}^{\mathrm{Cf}}} b_{k} w_{k}\right\|_{L^{2}[0,1]}^{2}\right)^{\frac{1}{2}} \\
& \leq\left(\left\|\sum_{k \in \mathcal{K}_{N}} a_{k}^{*} w_{k}\right\|_{L^{2}[0,1]}+\left\|\sum_{k \in \mathcal{K}_{N}} a_{k}^{*} w_{k}+\sum_{k \in \mathcal{K}_{N}^{\mathrm{Cf}}} b_{k} w_{k}\right\|_{L^{2}[0,1]} .\right.
\end{aligned}
$$

This shows that it is sufficient to consider the smaller feasible set

$$
\Gamma=\left\{\left\{b_{k}\right\}_{k \in \mathcal{K}_{N}^{\mathrm{Cf}}}:\left\|\left\{b_{k}\right\}_{k \in \mathcal{K}_{N}^{\mathrm{Cf}}}\right\|_{\ell^{2}\left(\mathcal{K}_{N}^{\mathrm{Cf}}\right)} \leq \sqrt{2}\right\}
$$

in the minimization problem. This is a finite dimensional set that is closed and bounded, and hence compact. It follows that the minimization problem

$$
\begin{aligned}
\left\{b_{k}^{*}\right\}_{k \in \mathcal{K}_{N}^{\mathrm{Cf}}} & =\underset{b \in \ell^{2}\left(\mathcal{K}_{N}^{\mathrm{Cf}}\right)}{\arg \min }\left\|\sum_{k \in \mathcal{K}_{N}} a_{k}^{*} w_{k}+\sum_{k \in \mathcal{K}_{N}^{\mathrm{Cf}}} b_{k} w_{k}\right\|_{L^{\infty}[0,1]} \\
& =\underset{b \in \Gamma}{\arg \min }\left\|\sum_{k \in \mathcal{K}_{N}} a_{k}^{*} w_{k}+\sum_{k \in \mathcal{K}_{N}^{\mathrm{Cf}}} b_{k} w_{k}\right\|_{L^{\infty}[0,1]}
\end{aligned}
$$

has a solution.

In order to generate the plot, we numerically solved the minimization problem (31) using CVXPY (version 1.0.6) in Python (version 3.5). Let

$$
s_{N}^{*}(t)=\sum_{k \in \mathcal{K}_{N}} a_{k}^{*} w_{k}(t)+\sum_{k \in \mathcal{K}_{N}^{\mathrm{Cf}}} b_{k}^{*} w_{k}(t)
$$

denote the compensated information signal, i.e., the transmit signal. In Figure 2, the values of $\left\|s_{N}^{*}\right\|_{L^{\infty}[0,1]}$ are plotted for $N=1, \ldots, 13$ in blue. It can be seen that the peak value of the transmit signal $\left\|s_{N}^{*}\right\|_{\infty}$ is smaller than or equal to $\sqrt{2}$ for all $N$.

Interestingly, for $N>2$, we have $\left\|s_{N}^{*}\right\|_{L^{\infty}[0,1]}<\sqrt{2}$, i.e., the peak value of the transmit signal is strictly smaller than $\sqrt{2}$. Hence, for the specific information sequence $\left\{a_{k}^{*}\right\}_{k \in \mathcal{K}_{N}}$ we achieve a better compensation than suggested by the smallest possible extension constant $\underline{C}_{\mathrm{EX}}\left(\mathcal{K}_{N}, 2^{N}\right)=\sqrt{2}$.

However, since according to Theorem $6 \underline{C}_{\mathrm{EX}}\left(\mathcal{K}_{N}, 2^{N}\right)=$ $\sqrt{2}$ for all $N \geq 2$, our theory shows that there must exist an information sequence $\left\{a_{k}^{* *}\right\}_{k \in \mathcal{K}_{N}}$ with $\left\|\left\{a_{k}^{* *}\right\}_{k \in \mathcal{K}_{N}}\right\|_{\ell^{2}\left(\mathcal{K}_{N}\right)}=$ 1 , for which we have

$$
\min _{b \in \ell^{2}\left(\mathcal{K}_{N}^{\mathrm{Cf}}\right)}\left\|\sum_{k \in \mathcal{K}_{N}} a_{k}^{* *} w_{k}+\sum_{k \in \mathcal{K}_{N}^{\mathrm{Cf}}} b_{k} w_{k}\right\|_{L^{\infty}[0,1]}=\sqrt{2} .
$$

Let $\mathcal{K}_{N} \subset[N]$ with $\left|\mathcal{K}_{N}\right| \geq 2$ and $k_{1}, k_{2} \in \mathcal{K}_{N}, k_{1} \neq k_{2}$, be arbitrary. Then such a signal is given by

$$
a_{k}^{* *}= \begin{cases}\frac{1}{\sqrt{2}}, & k \in\left\{k_{1}, k_{2}\right\}, \\ 0, & k \in \mathcal{K}_{N} \backslash\left\{k_{1}, k_{2}\right\} .\end{cases}
$$


Clearly we have $\left\|\left\{a_{k}^{* *}\right\}_{k \in \mathcal{K}_{N}}\right\|_{\ell^{2}\left(\mathcal{K}_{N}\right)}=1$. Interestingly, no compensation is required for this information sequence, since the compensation sequence $b_{K}^{* *}=0$ for all $k \in \mathcal{K}_{N}^{\mathrm{Cf}}$ is already optimal. We will prove this next by contradiction. As before, it is sufficient to use $\mathcal{K}_{N}^{\mathrm{Cf}}=\left[2^{N}\right] \backslash \mathcal{K}_{N}$ as a compensation set. Assume that there is a sequence $\left\{\tilde{b}_{k}\right\}_{k \in \mathcal{K}_{N}^{\mathrm{Cf}}}$ such that

$$
\left\|\frac{1}{\sqrt{2}} w_{k_{1}}+\frac{1}{\sqrt{2}} w_{k_{2}}+\sum_{k \in \mathcal{K}_{N}^{\text {Cf }}} b_{k} w_{k}\right\|_{L^{\infty}[0,1]}<\sqrt{2},
$$

and let

$$
\begin{aligned}
A=\int_{0}^{1} & \left(\frac{1}{\sqrt{2}} w_{k_{1}}(t)+\frac{1}{\sqrt{2}} w_{k_{2}}(t)\right) \\
& \times\left(\frac{1}{\sqrt{2}} w_{k_{1}}(t)+\frac{1}{\sqrt{2}} w_{k_{2}}(t)+\sum_{k \in \mathcal{K}_{N}^{\mathrm{Cf}}} b_{k} w_{k}(t)\right) \mathrm{d} t .
\end{aligned}
$$

Then we have

$$
\begin{aligned}
|A| \leq \int_{0}^{1} & \left|\frac{1}{\sqrt{2}} w_{k_{1}}(t)+\frac{1}{\sqrt{2}} w_{k_{2}}(t)\right| \\
& \times\left|\frac{1}{\sqrt{2}} w_{k_{1}}(t)+\frac{1}{\sqrt{2}} w_{k_{2}}(t)+\sum_{k \in \mathcal{K}_{N}^{\text {Cf }}} b_{k} w_{k}(t)\right| \mathrm{d} t \\
& <\sqrt{2} \int_{0}^{1}\left|\frac{1}{\sqrt{2}} w_{k_{1}}(t)+\frac{1}{\sqrt{2}} w_{k_{2}}(t)\right| \mathrm{d} t \\
& =1
\end{aligned}
$$

where we used (19) in the last line. We also have

$$
\begin{aligned}
A= & \int_{0}^{1}\left(\frac{1}{\sqrt{2}} w_{k_{1}}(t)+\frac{1}{\sqrt{2}} w_{k_{2}}(t)\right)^{2} \mathrm{~d} t \\
& +\int_{0}^{1}\left(\frac{1}{\sqrt{2}} w_{k_{1}}(t)+\frac{1}{\sqrt{2}} w_{k_{2}}(t)\right)\left(\sum_{k \in \mathcal{K}_{N}^{\mathrm{Cf}}} b_{k} w_{k}(t)\right) \mathrm{d} t .
\end{aligned}
$$

The second integral is zero because $\left\{w_{k}\right\}_{k \in \mathbb{N}}$ is an ONS, and for the first integral we obtain

$$
\int_{0}^{1}\left(\frac{1}{\sqrt{2}} w_{k_{1}}(t)+\frac{1}{\sqrt{2}} w_{k_{2}}(t)\right)^{2} \mathrm{~d} t=1,
$$

due to Parseval's identity. Hence, we see that $A=1$, which is a contradiction to (32).

\section{PAPR CONTROL FOR OFDM}

As in the CDMA case, the goal in the OFDM case is to answer the three questions that we presented in the introduction. It turns out that the analysis of OFDM is far more complicated than CDMA. We are only able to solve the problem for information sets $\mathcal{K}$ with $|\mathcal{K}|=2$. Nevertheless, the result is surprising, because for all $\mathcal{K}$ with $|\mathcal{K}|=2$ we have the same optimal extension constant $C_{\mathrm{EX}}^{\mathrm{OFDM}}(\mathcal{K})$, regardless of the specific elements of $\mathcal{K}$.

\section{A. Special Case: Information Sets of Cardinality Two}

Theorem 8. For OFDM, where the complete ONS is given by $\left\{\phi_{k}\right\}_{k \in \mathbb{Z}}=\left\{\mathrm{e}^{i 2 \pi k \cdot}\right\}_{k \in \mathbb{Z}}$, we have $\underline{C}_{E X}^{O F D M}(\mathcal{K})=\pi / \sqrt{8}$ for all $\mathcal{K} \subset \mathbb{Z}$ with $|\mathcal{K}|=2$.

Remark 4. It is interesting that for $|\mathcal{K}|=2$, the smallest possible extension constant for OFDM, which is $\pi / \sqrt{8} \approx 1.11$, is much smaller than the smallest possible extension constant for CDMA, which is $\sqrt{2} \approx 1.41$.

Remark 5. In the CDMA case we found that all optimal information sets $\mathcal{K}$ with $|\mathcal{K}|>2$ also achieve the same minimal extension constant $\sqrt{2}$. In general, the optimal extension constant is a non-decreasing function of $|\mathcal{K}|$. For OFDM we indeed suspect that the optimal extension constant increases with $|\mathcal{K}|$.

Proof of Theorem 8. Let $\mathcal{K}=\left\{k_{1}, k_{2}\right\} \subset \mathbb{Z}$ with $k_{1} \neq k_{2}$. According to Corollary 1 , we have

$$
\frac{1}{\underline{C}_{\mathrm{EX}}^{\mathrm{OFDM}}(\mathcal{K})}=\inf _{\substack{a_{1}, a_{2} \in \mathbb{C} \\\left|a_{1}\right|^{2}+\left|a_{2}\right|^{2}=1}} \int_{0}^{1}\left|a_{1} \mathrm{e}^{i 2 \pi k_{1} t}+a_{2} \mathrm{e}^{i 2 \pi k_{2} t}\right| \mathrm{d} t .
$$

Let $\phi_{1}=\arg \left(a_{1}\right)$ and $\phi_{2}=\arg \left(a_{2}\right)$. Since

$$
\begin{aligned}
& \int_{0}^{1}\left|a_{1} \mathrm{e}^{i 2 \pi k_{1} t}+a_{2} \mathrm{e}^{i 2 \pi k_{2} t}\right| \mathrm{d} t \\
& =\int_{0}^{1}|| a_{1}\left|\mathrm{e}^{i \phi_{1}} \mathrm{e}^{i 2 \pi k_{1} t}+\right| a_{2}\left|\mathrm{e}^{i \phi_{2}} \mathrm{e}^{i 2 \pi k_{2} t}\right| \mathrm{d} t \\
& =\int_{0}^{1}|| a_{1}|+| a_{2}\left|\mathrm{e}^{i\left[\phi_{2}-\phi_{1}+2 \pi t\left(k_{2}-k_{1}\right)\right]}\right| \mathrm{d} t \\
& =\frac{1}{2 \pi\left(k_{2}-k_{1}\right)} \int_{\phi_{2}-\phi_{1}}^{\phi_{2}-\phi_{1}+2 \pi\left(k_{2}-k_{1}\right)}|| a_{1}|+| a_{2}\left|\mathrm{e}^{i \tau}\right| \mathrm{d} \tau \\
& =\frac{1}{2 \pi} \int_{0}^{2 \pi}|| a_{1}|+| a_{2}\left|\mathrm{e}^{i \tau}\right| \mathrm{d} \tau \\
& =\frac{1}{\pi} \int_{0}^{\pi}|| a_{1}|+| a_{2}\left|\mathrm{e}^{i \tau}\right| \mathrm{d} \tau,
\end{aligned}
$$

where the second to last equality follows from the fact that ||$a_{1}|+| a_{2}\left|\mathrm{e}^{i \tau}\right|$ is a $2 \pi$-periodic function and the last equality follows out of symmetry reasons, we see that

$$
\frac{1}{\underline{C}_{\mathrm{EX}}^{\mathrm{OFDM}}(\mathcal{K})}=\inf _{\substack{u, v>0 \\ u^{2}+v^{2}=1}} \frac{1}{\pi} \int_{0}^{\pi}\left|u+v \mathrm{e}^{i \tau}\right| \mathrm{d} \tau .
$$

Let

$$
\Psi(u, v)=\frac{1}{\pi} \int_{0}^{\pi}\left|u+v \mathrm{e}^{i \tau}\right| \mathrm{d} \tau
$$

$\Psi(u, v)$ is a continuous function in $u$ and $v$. Hence, there exist $\hat{u}, \hat{v}$ with $\hat{u}, \hat{v}>0$ and $\hat{u}^{2}+\hat{v}^{2}=1$ such that

$$
\Psi(\hat{u}, \hat{v})=\inf _{\substack{u, v>0 \\ u^{2}+v^{2}=1}} \Psi(u, v)
$$


i.e., the infimum is in fact a minimum. For $u=\sqrt{\frac{1}{2}+h}$ and $v=\sqrt{\frac{1}{2}-h}$ with $h \in[-1 / 2,1 / 2]$, we therefore obtain

$$
\begin{aligned}
\Psi(u, v) & =\Psi\left(\sqrt{\frac{1}{2}+h}, \sqrt{\frac{1}{2}-h}\right) \\
& =\frac{1}{\pi} \int_{0}^{\pi}\left|\sqrt{\frac{1}{2}+h}+\sqrt{\frac{1}{2}-h} \mathrm{e}^{i \tau}\right| \mathrm{d} \tau \\
& =\frac{1}{\pi} \int_{0}^{\pi}\left(\frac{1}{2}+h+2 \sqrt{\frac{1}{2}-h} \sqrt{\frac{1}{2}-h} \cos \tau\right. \\
& \left.+\left(\frac{1}{2}-h\right) \cos ^{2}(\tau)+\left(\frac{1}{2}-h\right) \sin ^{2}(\tau)\right)^{\frac{1}{2}} \mathrm{~d} \tau \\
& =\frac{1}{\pi} \int_{0}^{\pi}\left(1+2 \sqrt{\frac{1}{4}-h^{2}} \cos \tau\right)^{\frac{1}{2}} \mathrm{~d} \tau \\
& =\frac{1}{\pi \sqrt{2}} \int_{0}^{\pi}\left(2+2 \sqrt{1-4 h^{2}} \cos \tau\right)^{\frac{1}{2}} \mathrm{~d} \tau .
\end{aligned}
$$

For $h \in[-1 / 2,1 / 2]$, we set

$$
F(h)=\frac{1}{\pi \sqrt{2}} \int_{0}^{\pi}\left(2+2 \sqrt{1-4 h^{2}} \cos \tau\right)^{\frac{1}{2}} \mathrm{~d} \tau .
$$

Clearly, we have $F(h)=F(-h)$. Hence, it suffices to consider $h \in[0,1 / 2]$ in the following. We have

$$
\begin{aligned}
F(0) & =\frac{1}{\pi \sqrt{2}} \int_{0}^{\pi} \sqrt{2+2 \cos (\tau)} \mathrm{d} \tau \\
& =\frac{2}{\pi \sqrt{2}} \int_{0}^{\pi} \cos (\tau / 2) \mathrm{d} \tau=\frac{\sqrt{8}}{\pi},
\end{aligned}
$$

and

$$
F(1 / 2)=\frac{1}{\pi \sqrt{2}} \int_{0}^{\pi} \sqrt{2} \mathrm{~d} \tau=1 .
$$

For $0<h<1 / 2$

$$
\left(2+2 \sqrt{1-4 h^{2}} \cos (\tau)\right)^{\frac{1}{2}}
$$

is infinitely often differentiable in $h$ and $\tau$, since

$$
2+2 \sqrt{1-4 h^{2}} \cos (\tau)>0
$$

for all $\tau \in[0, \pi]$. Hence, for $0<h<1 / 2$ we have

$$
\begin{aligned}
& F^{\prime}(h) \\
& \quad=\frac{1}{\pi \sqrt{2}} \int_{0}^{\pi} \frac{1}{2 \sqrt{2+2 \sqrt{1-4 h^{2}} \cos (\tau)}} \frac{-8 h \cos (\tau)}{\sqrt{1-4 h^{2}}} \mathrm{~d} \tau \\
& \quad=\frac{-4 h}{\pi \sqrt{2} \sqrt{1-4 h^{2}}} \int_{0}^{\pi} \frac{\cos (\tau)}{\sqrt{2+2 \sqrt{1-4 h^{2}} \cos (\tau)}} \mathrm{d} \tau .
\end{aligned}
$$

The function

$$
g_{h}(\tau)=\frac{1}{\sqrt{2+2 \sqrt{1-4 h^{2}} \cos (\tau)}}
$$

is monotonically increasing on $(0, \pi)$, i.e., we have $g_{h}^{\prime}(\tau)>0$ for all $\tau \in(0, \pi)$. We further have

$$
\begin{aligned}
\int_{0}^{\pi} g_{h}(\tau) \cos (\tau) \mathrm{d} \tau & =\left.g_{h}(\tau) \sin (\tau)\right|_{0} ^{\pi}-\int_{0}^{\pi} g_{h}^{\prime}(\tau) \sin (\tau) \mathrm{d} \tau \\
& =-\int_{0}^{\pi} g_{h}^{\prime}(\tau) \sin (\tau) \mathrm{d} \tau .
\end{aligned}
$$

Since $g_{h}^{\prime}(\tau)>0$ for all $\tau \in(0, \pi)$, it follows that

$$
\int_{0}^{\pi} g_{h}(\tau) \cos (\tau) \mathrm{d} \tau<0,
$$

and consequently that $F^{\prime}(h)>0$ for all $h \in(0,1 / 2)$. Thus, we see that

$$
\min _{h \in[0,1 / 2]} F(h)=F(0)=\frac{\sqrt{8}}{\pi},
$$

and obtain

$$
\min _{\substack{u, v>0 \\ u^{2}+v^{2}=1}} \Psi(u, v)=\frac{\sqrt{8}}{\pi} .
$$

From (33) and (34) it follows that $\underline{C}_{\mathrm{EX}}^{\mathrm{OFDM}}(\mathcal{K})=\pi / \sqrt{8}$.

The problem of calculating $\underline{C}_{\mathrm{EX}}^{\mathrm{OFDM}}(N)$ for $N \geq 3$ is completely open. Even bounds for $\underline{C}_{\mathrm{EX}}^{\mathrm{OFDM}}(N)$ seem to be unknown.

\section{B. Special Coefficient Sequences}

In the Walsh case, we succeeded in solving the optimization problem stated in (4), and thus were able to explicitly find optimal information sequences that achieve the minimal extension constant. For OFDM, the general problem is open. In the last subsection we were able to solve it for $|\mathcal{K}|=2$.

In this subsection we will discuss OFDM signals with specific coefficient sequences. For arbitrary $\mathcal{K}=\left\{k_{1}, \ldots, k_{N}\right\} \subset$ $\mathbb{Z}$ with $|\mathcal{K}|=N$, we consider the specific OFDM signals

$$
F_{\mathcal{K}}(t)=\sum_{k \in \mathcal{K}} \frac{1}{\sqrt{N}} \mathrm{e}^{i k t}
$$

where all information coefficients $a_{k}$ are equal, having the value $1 / \sqrt{N}$. Such OFDM signals recently garnered attention in the mathematical literature [37], [38]. In the following we will briefly analyze the connection between the $L^{1}$-norm of (35) and our PAPR problem. Formally, we see a resemblance between (35) and the sum (26) in the Walsh case. However, for OFDM, i.e., for (26), there is no theory analogous to the theory of perfect Walsh sums, which we employed in Section V-A.

Clearly, $F_{\mathcal{K}}$ is in the set

$$
\begin{aligned}
\mathfrak{F}^{1}(\mathcal{K})=\left\{f \in L^{1}[0,1]:\right. & \\
& \left.f=\sum_{k \in \mathcal{K}} a_{k} \mathrm{e}^{i 2 \pi k} \text { for some }\left\{a_{k}\right\}_{k \in \mathcal{K}} \subset \mathbb{C}\right\} .
\end{aligned}
$$

Let $\underline{C}_{\mathrm{EX}}^{\mathrm{OFDM}}(\mathcal{K})$ denote the optimal OFDM extension constant. From Corollary 1 we know that

$$
\frac{1}{\underline{C}_{\mathrm{EX}}^{\mathrm{OFDM}}(\mathcal{K})}=\inf _{\substack{f \in \mathfrak{F}^{1}(\mathcal{K}) \\\|f\|_{L^{2}[0,1]}=1}}\|f\|_{L^{1}[0,1]} .
$$


Hence, we have for all $\mathcal{K} \subset \mathbb{N}$ with $|\mathcal{K}|=N$ that

$$
\frac{1}{\underline{C}_{\mathrm{EX}}^{\mathrm{OFDM}}(\mathcal{K})} \leq\left\|F_{\mathcal{K}}\right\|_{L^{1}[-\pi, \pi]}
$$

Using the abbreviation

$$
\bar{C}(N)=\sup _{\substack{\mathcal{K} \subset \mathbb{N} \\|\mathcal{K}|=N}}\left\|F_{\mathcal{K}}\right\|_{L^{1}[-\pi, \pi]}
$$

we see that

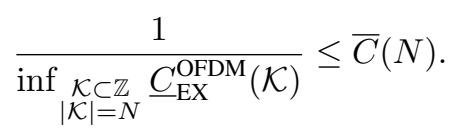

For $N=2$ we have shown in the previous subsection that

$$
\bar{C}(2)=\frac{1}{\inf _{\substack{\mathcal{K}|=2\\| \mathcal{K} \mid=2}} C_{\mathrm{EX}}^{\mathrm{OFDM}}(\mathcal{K})} .
$$

The results in [37], [38] show that

$$
\limsup _{N \rightarrow \infty} \bar{C}(N) \geq \frac{\sqrt{\pi}}{2} .
$$

Inspection of the calculations in [37], [38] and the resulting difficulties of solving the problem even for simple OFDM signals having the shape (35) strongly indicates that characterizing $C_{\mathrm{EX}}^{\mathrm{OFDM}}$ is a difficult task.

\section{CONCLUSION}

The control of the PAPR and finding optimal information sets is an important problem. In [39] it was shown for Walsh based CDMA systems that the information sets $\mathcal{K}$ for which the PAPR is solvable need to be sparse in a certain sense, in particular their upper densities need to be zero. However, no statement about the optimal information set was made. In general, little is known about the answers to questions $1-3$, and most of the results are based on simulations and not on analytic considerations. For our proof, the optimal constant in Khinchin's inequality [35], [36] was essential. It would also be interesting to answer the three questions for other orthogonal transmission schemes, e.g., OFDM, where the ONS is the system of complex exponentials. However, the present proof technique is tailored to the specific properties of the Walsh functions, and therefore cannot be used for other ONS.

In this paper, a purely deterministic analysis of the tone reservation method was performed. This approach is in the spirit of the original publications on tone reservation, [8] and [9]. Using mathematical methods from functional analysis, we were able to completely characterize the solvability of the PAPR problem - in this paper for CDMA and in [31] for general orthonormal transmission schemes-and to derive optimal constants.

Future research directions could comprise a stochastic analysis of the PAPR or a study of performance measures, such as the bit error rate. This path would be interesting not only from an application point of view but also for the further development of foundations of signal processing, to see if it is possible to connect probabilistic methods with functional analysis. Currently, it is not clear how the techniques from [31] can advance in this direction.

\section{ACKNOWLEDGMENT}

The first author wants to thank Bernd Haberland and Andreas Pascht, of Bell Labs, for bringing the PAPR problem for CDMA systems to his attention in 2000, and for discussions since that time. First results on the tone reservation problem for CDMA systems were presented at the Bell Labs Lecture of Holger Boche in 2010, where Bernd Haberland asked about the best performance that can be achieved with this method. Holger Boche also wants to thank Bernd Haberland for his hospitality at Bell Labs and the discussions during that time.

\section{REFERENCES}

[1] H. Boche and U. J. Mönich, "Optimal tone reservation for peak to average power control of CDMA systems," in Proceedings of the IEEE International Conference on Acoustics, Speech, and Signal Processing (ICASSP '18), Apr. 2018, pp. 3674-3678.

[2] L. Hanzo, L.-L. Yang, and K. Y. Ee-Lin Kuan, Single and Multi-Carrier DS-CDMA: Multi-User Detection, Space-Time Spreading, Synchronisation, Networking and Standards. Wiley-IEEE Press, 2003.

[3] S. Hara and R. Prasad, "Overview of multicarrier CDMA," IEEE Communications Magazine, vol. 35, no. 12, pp. 126-133, Dec. 1997.

[4] X. Li and L. J. Cimini, "Effects of clipping and filtering on the performance of OFDM," in 1997 IEEE 47th Vehicular Technology Conference. Technology in Motion, vol. 3, May 1997, pp. 1634-1638.

[5] S. Litsyn and A. Yudin, "Discrete and continuous maxima in multicarrier communication," IEEE Transactions on Information Theory, vol. 51, no. 3, pp. 919-928, Mar. 2005.

[6] S. Litsyn and G. Wunder, "Generalized bounds on the crest-factor distribution of OFDM signals with applications to code design," IEEE Transactions on Information Theory, vol. 52, no. 3, pp. 992-1006, Mar. 2006.

[7] G. Wunder, R. F. Fischer, H. Boche, S. Litsyn, and J.-S. No, "The PAPR problem in OFDM transmission: New directions for a long-lasting problem," IEEE Signal Processing Magazine, vol. 30, no. 6, pp. 130144, Nov. 2013.

[8] J. Tellado and J. M. Cioffi, "Efficient algorithms for reducing PAR in multicarrier systems," in Proceedings of the 1998 IEEE International Symposium on Information Theory, Aug. 1998, p. 191.

[9] - "Peak to average power ratio reduction," U.S.A. Patent 09/062, 867, Apr. 20, 1998

[10] K. G. Paterson and V. Tarokh, "On the existence and construction of good codes with low peak-to-average power ratios," IEEE Transactions on Information Theory, vol. 46, no. 6, pp. 1974-1987, Sep. 2000.

[11] S. Han and J. Lee, "An overview of peak-to-average power ratio reduction techniques for multicarrier transmission," IEEE Wireless Communications, vol. 12, no. 2, pp. 56-65, Apr. 2005.

[12] B. S. Krongold and D. L. Jones, "An active-set approach for OFDM PAR reduction via tone reservation," IEEE Transactions on Signal Processing, vol. 52, no. 2, pp. 495-509, Feb. 2004.

[13] A. Aggarwal and T. H. Meng, "Minimizing the peak-to-average power ratio of OFDM signals using convex optimization," IEEE Transactions on Signal Processing, vol. 54, no. 8, pp. 3099-3110, Aug. 2006.

[14] C.-L. Wang and Y. Ouyang, "Low-complexity selected mapping schemes for peak-to-average power ratio reduction in OFDM systems," IEEE Transactions on Signal Processing, vol. 53, no. 12, pp. 4652-4660, Dec. 2005.

[15] N. Chen and G. T. Zhou, "Superimposed training for OFDM: a peak-toaverage power ratio analysis," IEEE Transactions on Signal Processing, vol. 54, no. 6, pp. 2277-2287, Jun. 2006.

[16] G. T. Zhou and L. Peng, "Optimality condition for selected mapping in OFDM," IEEE Transactions on Signal Processing, vol. 54, no. 8, pp. 3159-3165, Aug. 2006.

[17] R. L. G. Cavalcante and I. Yamada, "A flexible peak-to-average power ratio reduction scheme for OFDM systems by the adaptive projected subgradient method," IEEE Transactions on Signal Processing, vol. 57, no. 4, pp. 1456-1468, Apr. 2009.

[18] C.-P. Li, S.-H. Wang, and C.-L. Wang, "Novel low-complexity SLM schemes for PAPR reduction in OFDM systems," IEEE Signal Processing Magazine, vol. 58, no. 5, pp. 2916-2921, May 2010.

[19] C. Siegl and R. F. H. Fischer, "Asymptotic performance analysis and successive selected mapping for PAR reduction in OFDM," IEEE Transactions on Signal Processing, vol. 58, no. 6, pp. 3228-3237, Jun. 2010. 
[20] M. S. Ahmed, S. Boussakta, B. S. Sharif, and C. C. Tsimenidis, "OFDM based on low complexity transform to increase multipath resilience and reduce PAPR," IEEE Transactions on Signal Processing, vol. 59, no. 12, pp. 5994-6007, Dec. 2011.

[21] H. Boche and B. Farrell, "PAPR and the density of information bearing signals in OFDM," EURASIP Journal on Advances in Signal Processing, vol. 2011, no. 1, pp. 1-9, 2011, invited paper.

[22] C. Li, T. Jiang, Y. Zhou, and H. Li, "A novel constellation reshaping method for PAPR reduction of OFDM signals," IEEE Transactions on Signal Processing, vol. 59, no. 6, pp. 2710-2719, Jun. 2011.

[23] S.-S. Eom, H. Nam, and Y.-C. Ko, "Low-complexity PAPR reduction scheme without side information for OFDM systems," IEEE Transactions on Signal Processing, vol. 60, no. 7, pp. 3657-3669, Jul. 2012.

[24] T. Jiang, C. Ni, D. Qu, and C. Wang, "Energy-efficient NCOFDM/OQAM-based cognitive radio networks," IEEE Communications Magazine, vol. 52, no. 7, pp. 54-60, Jul. 2014.

[25] S.-H. Wang, C.-P. Li, K.-C. Lee, and H.-J. Su, "A novel low-complexity precoded OFDM system with reduced PAPR," IEEE Transactions on Signal Processing, vol. 63, no. 6, pp. 1366-1376, Mar. 2015.

[26] T. Jiang, C. Ni, C. Ye, Y. Wu, and K. Luo, "A novel multi-block tone reservation scheme for PAPR reduction in OQAM-OFDM systems," IEEE Transactions on Broadcasting, vol. 61, no. 4, pp. 717-722, Dec. 2015.

[27] C. Ni, Y. Ma, and T. Jiang, "A novel adaptive tone reservation scheme for PAPR reduction in large-scale multi-user MIMO-OFDM systems," IEEE Wireless Communications Letters, vol. 5, no. 5, pp. 480-483, Oct. 2016.

[28] J. Tellado, Multicarrier Modulation with Low PAR: Applications to DSL and Wireless. Kluwer Academic Publishers, 2000.

[29] H. Boche, U. J. Mönich, and E. Tampubolon, "Complete characterization of the solvability of PAPR reduction for OFDM by tone reservation," in Proceedings of the 2017 IEEE International Symposium on Information Theory, Jun. 2017, pp. 2023-2027.

[30] H. Boche and U. J. Mönich, "Tone reservation and solvability concepts for the PAPR problem in general orthonormal transmission systems," in Proceedings of the IEEE International Conference on Acoustics, Speech, and Signal Processing (ICASSP '18), Apr. 2018, pp. 3669-3673.

[31] _ - "Peak to average power control via tone reservation in general orthonormal transmission systems," IEEE Transactions on Signal Processing, vol. 66, no. 13, pp. 3520-3528, Jul. 2018.

[32] N. J. Fine, "On the Walsh functions," Transactions of the American Mathematical Society, vol. 65, pp. 372-414, May 1949.

[33] H. Boche and V. Pohl, "Signal representation and approximationfundamental limits," European Transactions on Telecommunications (ETT), Special Issue on Turbo Coding 2006, vol. 18, no. 5, pp. 445-456, 2007.

[34] H. Boche and E. Tampubolon, "Mathematics of signal design for communication systems," in Mathematics and Society, W. König, Ed. European Mathematical Society Publishing House, 2016, pp. 185-220.

[35] S. J. Szarek, "On the best constants in the Khinchin inequality," Studia Mathematica, vol. 58, no. 2, pp. 197-208, 1976.

[36] U. Haagerup, "The best constants in the Khintchine inequality," Studia Mathematica, vol. 70, no. 3, pp. 231-283, 1981. [Online]. Available: http://eudml.org/doc/218383

[37] C. Aistleitner, "On a problem of Bourgain concerning the $l^{1}$-norm of exponential sums," Mathematische Zeitschrift, vol. 275, no. 3, pp. 681688, Dec. 2013

[38] S. Choi and T. Erdélyi, "On a problem of Bourgain concerning the $l_{p}$ norms of exponential sums," Mathematische Zeitschrift, vol. 279, no. 1-2, pp. 577-584, Feb. 2015.

[39] H. Boche and E. Tampubolon, "Asymptotic analysis of tone reservation method for the PAPR reduction of CDMA systems," in Proceedings of the 2017 IEEE International Symposium on Information Theory, 2017, pp. 2723-2727.

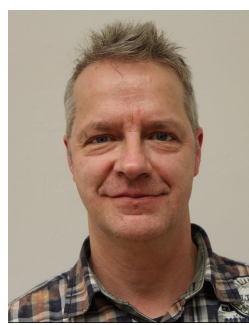

Holger Boche (M'04-SM'07-F'11) received the Dipl.-Ing. and Dr.-Ing. degrees in electrical engineering from the Technische Universität Dresden, Dresden, Germany, in 1990 and 1994, respectively. He graduated in mathematics from the Technische Universität Dresden in 1992. From 1994 to 1997, he did Postgraduate studies in mathematics at the Friedrich-Schiller Universität Jena, Jena, Germany. $\mathrm{He}$ received his Dr. rer. nat. degree in pure mathematics from the Technische Universität Berlin, Berlin, Germany, in 1998. In 1997, he joined the Heinrich-Hertz-Institut (HHI) für Nachrichtentechnik Berlin, Berlin, Germany. Starting in 2002, he was a Full Professor for mobile communication networks with the Institute for Communications Systems, Technische Universität Berlin. In 2003, he became Director of the Fraunhofer German-Sino Lab for Mobile Communications, Berlin, Germany, and in 2004 he became the Director of the Fraunhofer Institute for Telecommunications (HHI), Berlin, Germany. Since October 2010 he has been with the Institute of Theoretical Information Technology and Full Professor at the Technische Universität München, Munich, Germany. Since 2014 he has been a member and honorary fellow of the TUM Institute for Advanced Study, Munich, Germany. He was a Visiting Professor with the ETH Zurich, Zurich, Switzerland, during the 2004 and 2006 Winter terms, and with KTH Stockholm, Stockholm, Sweden, during the 2005 Summer term. Prof. Boche is a Member of IEEE Signal Processing Society SPCOM and SPTM Technical Committee. He was elected a Member of the German Academy of Sciences (Leopoldina) in 2008 and of the Berlin Brandenburg Academy of Sciences and Humanities in 2009. He received the Research Award "Technische Kommunikation" from the Alcatel SEL Foundation in October 2003, the "Innovation Award" from the Vodafone Foundation in June 2006, and the Gottfried Wilhelm Leibniz Prize from the Deutsche Forschungsgemeinschaft (German Research Foundation) in 2008. He was co-recipient of the 2006 IEEE Signal Processing Society Best Paper Award and recipient of the 2007 IEEE Signal Processing Society Best Paper Award. He was the General Chair of the Symposium on Information Theoretic Approaches to Security and Privacy at IEEE GlobalSIP 2016. Among his publications is the recent book Information Theoretic Security and Privacy of Information Systems (Cambridge University Press)

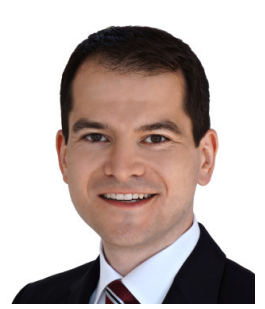

Ullrich J. Mönich (S'06-M'12-SM'16) received the Dipl.-Ing. degree in electrical engineering from the Technische Universität Berlin, Berlin, Germany, in 2005 and the Dr.-Ing. degree from the Technische Universität München, Munich, Germany, in 2011 During the winter term of 2003 , he was a visiting researcher with the University of California, Santa Barbara. From 2005 to 2010 he was Research and Teaching Assistant with the Technische Universität Berlin and from 2010 to 2012 with the Technische Universität München. From 2012 to 2015 he was a Postdoctoral Fellow at the Massachusetts Institute of Technology. Since 2015 he has been a Senior Researcher and Lecturer with the Technische Universität München. His research activities comprise signal and sampling theory, applied functional and harmonic analysis, and forensic DNA signal analysis. He was recipient of the Rohde \& Schwarz Award for his dissertation in 2012. 